\title{
The Confirmation of Hypothesis of the Absolute Reference System
}

\author{
Konstantinos Patrinos \\ National Technical University of Athens, Athens, Greece \\ Email:kpatr@central.ntua.gr
}

How to cite this paper: Patrinos, K. (2020) The Confirmation of Hypothesis of the Absolute Reference System. Journal of Applied Mathematics and Physics, 8, 999-1015. https://doi.org/10.4236/jamp.2020.85078

Received: April 28, 2020

Accepted: May 29, 2020

Published: June 2, 2020

Copyright $\odot 2020$ by author(s) and Scientific Research Publishing Inc. This work is licensed under the Creative Commons Attribution International License (CC BY 4.0).

http://creativecommons.org/licenses/by/4.0/ (c) (i) Open Access

\begin{abstract}
The purpose of the research in this article is the examination of the agreement of the hypothesis of the absolute reference system with the results of experiments that have been implemented in the past in order to confirm the special theory of relativity. To achieve this goal, we have chosen for discussing a theoretical topic of electromagnetism, that of electromagnetic mass calculation, and some experiments, some of which concern the transverse Doppler effect in a rotated system, two experiments that concern the kinetic energy measurement of accelerated electrons, one of which is the well known Bertozzis experiment, one experiment that concerns the propagation of Coulomb fields and one more experiment that concerns the effect of annihilation. The basic principles of the hypothesis of the absolute reference system, and the electromagnetic theory derived from these principles, are used to explain the experimental results. In these examples, the hypothesis of the absolute reference system is confirmed, since the experimental results agree with the predictions of this hypothesis. Also, in the discussion of calculation of electromagnetic mass is addressed the difficulty of solving this problem, when someone tries to solve this according to the energy-mass relation of the theory of relativity.
\end{abstract}

\section{Keywords}

Absolute Reference System, Electromagnetic Mass, Transverse Doppler Effect, Kinetic Energy of Accelerated Electrons

\section{Introduction}

In all the experiments carried out in order to examine the agreement of the special theory of relativity with the corresponding experimental results, the theoretical predictions that are commented on in relation to this agreement are those of the theory of relativity and the Newtonian physics. This means that the theories 
examined in all these experiments are the special theory of relativity and Newtonian physics. Bertozzi's experiment is one such example. What is being proven in this experiment is that the speed of the accelerating electrons can never reach the speed of light in vacuum. However, as can be seen from a closer look at this article, the expected relativistic kinetic energy values resulting from the measured velocities of the electrons deviate greatly from the measured kinetic energies, which were measured by a thermodynamic method. But because this deviation for the corresponding Newtonian energies was much greater, this was enough to convince the scientific community of the correctness of the theory of special relativity.

Of course, Newtonian physics deviates significantly from experimental reality as particle speed and energy increase. This article examines a new theory in terms of its agreement with these experimental results, and this theory is expressed in the newly introduced hypothesis of the absolute reference system. The previous comparison, therefore, concerns in this case the predictions of the special theory of relativity in comparison with the predictions of the hypothesis of the absolute reference system, regarding these experimental results.

It was deemed appropriate in this article to first consider a theoretical issue, that of the problem of incompatibility of the electromagnetic mass with the relation $E=m c^{2}$ of the special theory of relativity, because this incompatibility is eliminated by applying the principles of hypothesis of the absolute reference system for the electromagnetic interactions. This is a much-discussed problem, but we will refer specifically to the relevant analysis of this issue by Feynman, as it is examined in [1], and in this analysis also are presented the efforts to solve this problem. Despite these efforts, however, the relativistic problem remains, as one can see by reading the current literature.

In the study of the experiment of positron-electron annihilation process, it seems that the theoretical results of the hypothesis of the absolute reference system are the same as those of the special relativity. In all other experiments studied in this article, the agreement of the newly introduced hypothesis with the experimental results is examined, because there is no such agreement of the experimental results and the corresponding relativistic theoretical predictions (for example, the experiment for Measuring Propagation Speed of Coulomb Fields, in [2]).

The attempt to interpret natural phenomena on the basis of the hypothesis of an absolute reference system does not based on the attempt to interpret the phenomena using the relativistic conception, which was formulated in Galilean relativity and subsequently in Einstein's theory of relativity. The relativistic notion is based on the evaluation of physical quantities by the observer of the natural phenomenon at the observation point. On the basis of the absolute reference system, the space-time is Newtonian and the physical quantities such as momentum, force and kinetic energy are not determined on the basis of the relative velocity of the observed object by the observer's reference system, but on the basis of the object's reference system and the inertial reference system of the 
source of the field that affects the state of the object.

Electromagnetic interactions based on this hypothesis are carried out by force carriers, which are real photons with some peculiar behavior, unknown to this day, although this theoretical result is derived from classical electromagnetic theory. Also as explained in [3] the origin of creation of mass is electromagnetic and the same is true for the origin of the quantum nature of matter particles, as described in detail in [4]. Using this hypothesis, we will then provide answers to unresolved, by the modern physics theory, issues.

\section{Electromagnetic Mass}

The very old problem of electromagnetic mass in relation to the equation of mass and energy in the special theory of relativity is the first issue that we will consider in this paper. We will begin first with the study of this subject according to classical electromagnetic theory.

We consider a particle of charge $e$ moving at velocity $v$ with respect to the inertial laboratory reference system. We will calculate the energy of the electromagnetic field in space between two concentric spheres of radii $a$ and $b$. We consider that the center of the spheres is the center of mass of the charged particle. We will then calculate the momentum and mass of the electromagnetic field in the space between the two spheres ${ }^{1}$. Assuming $a<b$ the electromagnetic energy is:

$$
U_{\text {elec }}=\frac{1}{2} \frac{e^{2}}{4 \pi \epsilon_{o}}\left(\frac{1}{a}-\frac{1}{b}\right)
$$

when the speed $\boldsymbol{v}$ is much less than the speed of light in the vacuum, the momentum of that field is:

$$
\boldsymbol{p}=\frac{2}{3} \frac{e^{2}}{4 \pi \epsilon_{o} c^{2}} \boldsymbol{v}\left(\frac{1}{a}-\frac{1}{b}\right)
$$

The corresponding electromagnetic mass is:

$$
m_{\text {elec }}=\frac{2}{3} \frac{e^{2}}{4 \pi \epsilon_{o} c^{2}}\left(\frac{1}{a}-\frac{1}{b}\right)=\frac{4}{3} \frac{U_{\text {elec }}}{c^{2}}
$$

According to the absolute reference system hypothesis the previous equation is fully justified. It turns out that, on the basis of this hypothesis, the force carriers of Coulomb electric field are real photons. It also turns out that part of the energy $\hbar \omega$ of these photons is the energy of an elementary mass. The amount of energy of this elemental mass, as shown by the relevant calculations ${ }^{2}$, is equal to $\frac{2}{3} \hbar \omega$. If we denote by $m_{p h}$ the mass of this photon, the energy equation of this photonic mass is $m_{p h} c^{2}=\frac{2}{3} \hbar \omega$.

This result has been obtained by making use of Maxwell's equations and the

${ }^{1}$ The solution to this problem is analogous to that in [1], section 28 .

${ }^{2}$ [3], subsection 2.4 (equations 2.44). 
Coulomb field spherical symmetry. Also, according to the same hypothesis, the total kinetic energy of all the photons of the field in the space between the two spheres, for $N$ photons, is $U_{\text {elec }}=\frac{1}{2} \hbar \sum_{i=1}^{N} \omega_{i}$. Therefore, in accordance with all the foregoing, which derive on the basis of the absolute reference system hypothesis, the electromagnetic mass is:

$$
m_{\text {elec }}=\sum_{i=1}^{N} m_{\text {phi }}=\frac{2}{3} \frac{\hbar}{c^{2}} \sum_{i=1}^{N} \omega_{i}=\frac{4}{3} \frac{U_{\text {elec }}}{c^{2}}
$$

\section{Transverse Doppler Effect in a Rotated System}

The origin of this problem lies in Walter Kundig's 1963 experiment entitled "Measurement of the Transverse Doppler Effect in an Accelerated System", in [5]. A re-analysis of the experimental data of this scientific work, by Alexander L Kholmetskii, Tolga Yarman, Oleg V Missevitch and Boris I Rogozev, in 2008, showed that they conclude that "instead of the relative energy shift $\Delta E / E=-(1.0065 \pm 0.011) v^{2} / 2 c^{2}$ reported by Kundig ( $v$ being the linear velocity of absorber and $c$ being the light velocity in vacuum), we derive from his results $\Delta E / E=-(1.192 \pm 0.011) v^{2} / 2 c^{2}$ " and the details of this data analysis are in [6].

The experiment was repeated in 2009 by the same scientific team, and the result of the experiment, exposed to [7], showed that the results of the previous analysis are confirmed by the new experimental results. In particular, as a result, for the equation $\Delta E / E=-k v^{2} / c^{2}$, they obtained the overall estimation $k=0.68 \pm 0.03$.

One attempt to explain this experimental result on the basis of theory of relativity, in 2015, in [8] was unsuccessful, as detailed in [9], in the response given by the aforementioned authors.

The experiment has been repeated several times. In 2011 by A. L. Kholmetskii, T. Yarman and O. V. Missevitch. (Int. J. Phys. Sci. 6, 84 (2011)), the experimental result yields the coefficient $k=0.66 \pm 0.03$ within the expression frame for the relative energy shift between emission and absorption lines $\Delta E / E=-k v^{2} / c^{2}$.

We will then use the theory of the absolute reference system to address this problem. According to this hypothesis in the Coulomb field of an atomic nucleus of the absorber material, the energy $\hbar \omega$ of the absorbed photons is divided into two components of energy, due to the two components of their helical motion within this field, as explained in [3]. This is due, as we have already mentioned, to the spherical symmetry of the electric field of the nucleus and to the wave equation of the electric field, which results from Maxwell's equations. One of the two components of the motion is radial and if the corresponding angular frequency is denoted by $\omega_{r}$, the corresponding energy is equal to $\hbar \omega_{r}=\frac{1}{3} \hbar \omega$. The other component is circular and the corresponding energy, characterized as the energy of mass of the photon, is equal to $\hbar \omega_{v}=\frac{2}{3} \hbar \omega$. 
In the case of the experiment in [5] the absorber rotates at a speed $u$. The rate of energy absorption of the radial component of the motion of the photons is determined by the Doppler effect ${ }^{3}$. So the corresponding absorbed energy is estimated from the relation $\hbar \omega_{r}^{\prime}=\gamma \hbar \omega_{r}$, where $\gamma=\left(1-u^{2} / c^{2}\right)^{-1 / 2}$ and the angular frequency measured by the clock of the momentarily inertial reference system of absorber is denoted by $\omega_{r}^{\prime}$. Also we denote by $\omega_{r}$ the angular frequency measured by the clock of the laboratory reference system. The energy supplied by the source, due to the time contraction, will be $\gamma$ times less than that measured by the clock of the absorber, and are given by the relation:

$$
E_{a r}=\frac{1}{\gamma} \hbar \omega_{r}^{\prime}=\hbar \omega_{r}
$$

The amount of energy $\hbar \omega_{v}$, characterized as energy of mass, obeys the kinetic energy conservation relation of an elementary photon mass in the source-absorber system:

$$
\frac{1}{2} \hbar \omega_{v}=\frac{1}{2} \hbar \omega_{v a}+E_{p h k i n}
$$

where $E_{\text {phkin }}$ is the kinetic energy of the photonic mass due to the rotary motion of the absorber and the angular frequency of the photonic mass, measured by the clock of the instantaneous inertial system of the absorber, is denoted by $\omega_{v a}$. If the absorbed photon mass is denoted by $m_{v a}$, the previous relation becomes:

$$
\begin{aligned}
\frac{1}{2} \hbar \omega_{v} & =\frac{1}{2} m_{v a} c^{2}+\frac{1}{2} m_{v a} \gamma^{2} u^{2} \\
& =\frac{1}{2} m_{v a} c^{2}\left(1+\gamma^{2} \beta^{2}\right) \\
& =\frac{1}{2} \gamma^{2} \hbar \omega_{v a}
\end{aligned}
$$

where $m_{v a}=\hbar \omega_{v a} / c^{2}$ and $\beta=u / c$. The angular frequency of the absorbed mass according to the last relation is $\omega_{v a}=\omega_{v} / \gamma^{2}$. The change in absorption rate due to the Doppler effect is canceled by the time contraction as in the case of calculating the radial motion energy $\hbar \omega_{r}$, previously mentioned. The energy supplied by the source will be given by the relation:

$$
E_{a v}=\hbar \omega_{v a}=\frac{1}{\gamma^{2}} \hbar \omega_{v}
$$

Therefore, the total absorbed photon energy is $E_{a}=E_{a r}+E_{a v}$, while the total energy of a source photon is $E_{s}=\hbar \omega$ and only an amount of kinetic energy, equal to $E_{p h k i n}=\frac{1}{2} m_{v a} \gamma^{2} u^{2}$, is offered by the rotated absorber, whereby, the equation of the relative energy shift between emission and absorption lines, is:

${ }^{3}$ The rate of absorption of the photons results from the relation (4.31) of section 4.2, Doppler Effect, in [3], in the inertial reference system of the laboratory instead of the absolute reference system, where we set $u_{\Gamma}=0, \phi=0, c_{g}=c, u_{g}=u, \gamma_{g}=\gamma=\left(1-u^{2} / c^{2}\right)^{-1 / 2}$ and the frequencies $v_{g}$ and $v_{\Gamma}$ are replaced by the angular frequencies $\omega_{r}^{\prime}$ and $\omega_{r}$ according to clock of the absorber and the laboratory clock respectively. 


$$
\begin{aligned}
\frac{E_{a}-E_{s}}{E_{s}} & =\frac{\hbar \omega_{r}+\frac{1}{\gamma^{2}} \hbar \omega_{v}-\hbar \omega}{\hbar \omega} \\
& =\frac{\frac{1}{3} \hbar \omega+\frac{1}{\gamma^{2}} \frac{2}{3} \hbar \omega-\hbar \omega}{\hbar \omega} \\
& =-\frac{2}{3} \beta^{2}
\end{aligned}
$$

This result is in excellent agreement with the above experimental results.

\section{Kinetic Energy Measurement of Accelerated Electrons}

In order to experimentally test the correctness of the absolute reference system hypothesis, we will study two experiments measuring the kinetic energy of electrons, which simultaneously measure the beam electron velocity in a linear accelerator. The peculiarity of these experiments is that the velocity is measured by measuring the flight time of the electrons at a given travel length. One of these two experiments is the historical experiment of Bertozzi, described in [10]. The other experiment is that of M. Lund and U. I. Uggerhøj and the relevant details and experimental results are reported in [11].

According to the absolute reference system hypothesis, kinetic energy which is transferred to the detection device, which is the target of accelerated electrons, when the kinetic energy $E_{a}$ of the electrons is high enough so that the scattering of the force carriers by atoms of target to be no longer elastic, equals $E_{a} / 2$. The remaining half of the kinetic energy of the electrons is converted into mass absorbed by the atoms of the target. This absorbed mass is derived from the photon mass of the electromagnetic field force carriers of the accelerated electrons. As we have mentioned, this electromagnetic mass of the force carriers are real photons that exhibit such behavior under the given conditions in the Coulomb field.

A first estimate of this phenomenon in the Bertozzi experiment is made by studying the experimental results presented in [4]. There are two very useful measurements of the detectable kinetic energy of the electrons in this experiment, derived from the corresponding measurements of the target heat gain by simultaneously measuring the charge, that is, the number of beam electrons that hit the target. These measurements are shown in Table 1.

Table 1. Experimental results of W. Bertozzi's measurements, as set out in his work entitled “The Ultimate Speed", in 1964. The measured increase of the target heat per electron is denoted by $E_{m}$.

\begin{tabular}{cccc}
\hline $\begin{array}{c}\text { Measured } \\
\text { energy } \\
E_{m}, \mathrm{MeV}\end{array}$ & $\begin{array}{c}\text { Electron } \\
\text { velocity } \\
u, \times 10^{8} \mathrm{~m} / \mathrm{sec}\end{array}$ & $\begin{array}{c}\text { Relativistic } \\
\text { kin. energy } \\
E_{k}, \mathrm{MeV}\end{array}$ & $\begin{array}{c}\text { Detectable } \\
\text { kin. energy } \\
E_{\text {dee }}, \mathrm{MeV}\end{array}$ \\
\hline 1.6 & 2.88 & 1.3 & 1.5 \\
4.8 & 2.96 & 2.7 & 4.8 \\
\hline
\end{tabular}


The transmitted kinetic energy of the beam is equal to the increase in target heat. This energy per electron, measured by the thermal arrangement of the Bertozzi experiment, is denoted by $E_{m}$ in Table 1 . The relativistic kinetic energy calculated from the measured velocity $u$ is denoted by $E_{k}$ and is equal to $m(\gamma-1) c^{2}$, where $\gamma=\left(1-u^{2} / c^{2}\right)^{-1 / 2}$ and $m$ is the mass of the electron. The corresponding kinetic energy transferred according to the absolute reference system hypothesis is $E_{\text {det }}=\frac{1}{4} m \gamma^{2} u^{2}$, while the kinetic energy of the beam electrons is $E_{a}=\frac{1}{2} m \gamma^{2} u^{2}$. The values of these kinetic energies are in Table 1, where it appears that the energy values $E_{\text {det }}$, predicted by the absolute reference system hypothesis, are in excellent agreement with the measured values $E_{m}$.

The transfer of kinetic energy in the form of heat to the target is accomplished by the transfer of the kinetic energy of the electromagnetic mass of the force carriers of the electron beam electromagnetic field of the linear accelerator. The electromagnetic mass $m_{e l}$, passing through the active scattering cross section of the force carriers with the bound electron of an atom in the target material, transfers all the kinetic energy of the beam electron and is equal to the mass of this electron from which it is derived, that is, $m=m_{e l}$. In order for the scattering to be completely inelastic, it must be possible to incorporate half the kinetic energy of the beam's electron, that is energy equal to $E_{a} / 2$, as photonic mass in the atoms of the target material. There is a limitation to be implemented this completely inelastic interaction. Assuming that $N$ force carriers transfer the kinetic energy of a beam electron, the corresponding electromagnetic mass, according to the relation (4), is $m_{e l}=\frac{2}{3} \frac{\hbar}{c^{2}} \sum_{i=1}^{N} \omega_{i}$. Therefore for fully inelastic scattering the energy $E_{a} / 2$ should be greater than $\hbar \sum_{i=1}^{N} \omega_{i}$, which is the total energy of the Coulomb field in the area of the specific electromagnetic mass $m_{e l}$. Therefore the threshold for a fully inelastic scattering is determined by the relation:

$$
\frac{E_{a}}{2}=\frac{1}{4}\left(\gamma^{2}-1\right) m c^{2}=\hbar \sum_{i=1}^{N} \omega_{i}=\frac{3}{2} m_{e l} c^{2}
$$

Since $m=m_{e l}$, the last relation gives $(1 / 4)\left(\gamma^{2}-1\right)=3 / 2$ and therefore this relation is satisfied for $\gamma$ values greater than a critical value of $\gamma$ which is $\gamma_{c}=\sqrt{7}$ and a corresponding critical speed $u_{c}$ given by the relation $u_{c}=\sqrt{\frac{6}{7}} c$. The corresponding kinetic energy is $E_{c}=\frac{1}{4}\left(\gamma_{c}^{2}-1\right) m c^{2}=\frac{3}{2} m c^{2}$. Therefore the criterion for completely inelastic scattering is $\gamma \geq \sqrt{7}$ or equivalently $\frac{1}{2} E_{a} \geq \frac{3}{2} m c^{2}$.

For $\gamma<\sqrt{7}$ an amount of energy equal to $\frac{1}{4}\left(\gamma^{2}-1\right) m c^{2}$ is not sufficient to fully incorporate all the electromagnetic mass $m_{e l}$, but only one part of it, which 
is equal to $a m_{e l}$, where the coefficient $a$ is equal to the ratio of the energy values $\left(E_{a} / 2\right) / E_{c}$, that is, $a=\left(\gamma^{2}-1\right) /\left(\gamma_{c}^{2}-1\right)$. In this case the amount of kinetic energy incorporated as a mass is equal to $\frac{1}{4}\left(\gamma^{2}-1\right) a m_{e l} c^{2}$. Therefore the detectable kinetic energy transferred to the target will be:

$$
\begin{aligned}
E_{\text {det }} & =\frac{1}{2}\left(\gamma^{2}-1\right) m c^{2}-\frac{1}{4}\left(\gamma^{2}-1\right) \frac{\gamma^{2}-1}{6} m c^{2} \\
& =\left(\frac{\gamma^{2}-1}{2}-\frac{\left(\gamma^{2}-1\right)^{2}}{24}\right) m c^{2}
\end{aligned}
$$

According to the latter relation, at low speeds, that is, $u \ll c$ and $\gamma \rightarrow 1$, the detectable value of energy tends to the Newtonian value of kinetic energy, that is, $E_{\text {det }} \rightarrow \frac{1}{2} m u^{2}$.

The electron beam measured energy values of the experiment by M. Lund and U. I. Uggerhøj are in excellent agreement with the values of the energy curve $E_{\text {det }}$ in the two aforementioned speed ranges, that is, for $\gamma \geq \sqrt{7}$, where $E_{\text {det }}=E_{a} / 2$, and for $\gamma \leq \sqrt{7}$, where the relation (11) applies, that is, the experimental data in [10] and [11] (see Figure 1) are in excellent agreement with the detectable energy curves $E_{\text {det }}=\frac{1}{4}\left(\gamma^{2}-1\right) m c^{2}$ for $\frac{u}{c} \geq \sqrt{\frac{6}{7}}$ and $E_{\text {det }}=\left(\left(\gamma^{2}-1\right) / 2-\left(\gamma^{2}-1\right)^{2} / 24\right) m c^{2}$ for $\frac{u}{c} \leq \sqrt{\frac{6}{7}}$.

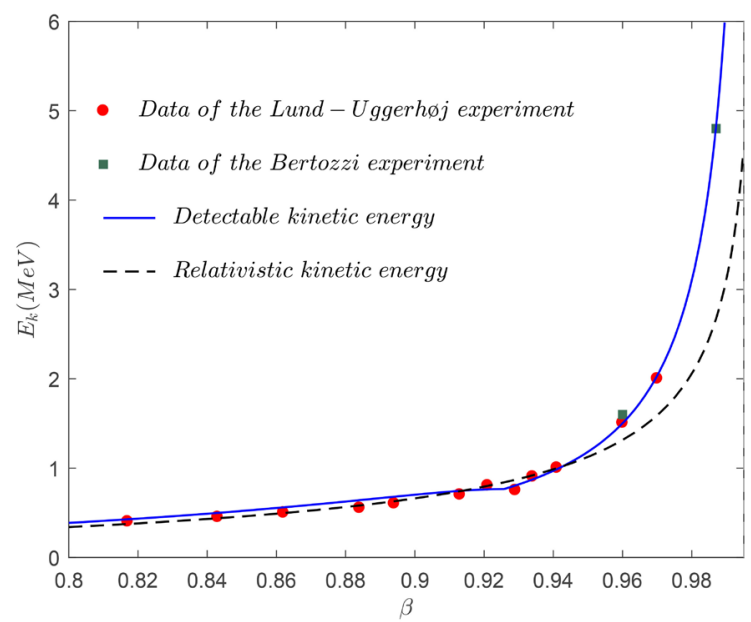

Figure 1. The kinetic energy of the electron obtained with $0.42 \mathrm{MeV}$ electrons from a linear accelerator as a function of the ratio of the electron velocity and the speed of light $c$. The continuous line is a curve based on the expression of detectable energy in region $\frac{u}{c} \geq \sqrt{\frac{6}{7}}$ where the equation of detectable energy is $E_{\text {det }}=\frac{1}{4}\left(\gamma^{2}-1\right) m c^{2}$ and $\frac{u}{c} \leq \sqrt{\frac{6}{7}}$ where the relation $E_{\text {det }}=\left(\left(\gamma^{2}-1\right) / 2-\left(\gamma^{2}-1\right)^{2} / 24\right) m c^{2}$ holds. The dashed line is a curve based on the relativistic expression, $E_{k}=m(\gamma-1) c^{2}$. 
In the case of very high energies of electrons, for example energies of the order of several tens of $\mathrm{TeV}$, due to the very high value of the coefficient $\gamma$, the actual physical contraction of the particle contributes to the decomposition of the particle, and, so, the particle no longer behaves as a solid body. This has the effect of hitting the target by a photon beam instead of the particle beam and these photons of the total mass of the particles have frequencies denoted by $v_{i l}$, measured in the laboratory, which are $\gamma$ times smaller than the corresponding measured frequencies in the particle reference system.

It should be noted that according to the hypothesis of the absolute reference system, the Lorentz contraction is a real physical contraction and not a geometric contraction, in Minkowski's space-time, which concerns the observer's inertial system, as inferred from the special theory of relativity.

In this case the kinetic energy is $E_{a}=(1 / 2) \gamma h \sum_{i} v_{i l}=(1 / 2)\left(\gamma^{2}-1\right) m c^{2}$ and the total energy transferred to the target is equal to $h \sum_{i} v_{i l} \simeq m \gamma c^{2}$.

\section{Propagation of Coulomb Fields}

The theoretical calculations in this section concern the measurement of the potential difference that results from the effect of the maximum transverse electric field on the detector of the experiment in [2]. The detection device comprises a Data Acquisition System described as "Data Acquisition System by means of fast, terminated coax cables. To record the sensors waveforms we used a Switched Capacitor Array (SCA) circuit (CAEN mod V1472) able to sample the input signal at $5 \mathrm{GHz}$. In addition to the sensors output, the SCA stored also the LINAC-RF trigger and the toroid pulse ..."

According to the following equation ([12], page 664, relation (14.14)):

$$
[\boldsymbol{E}]_{r e t}=\frac{e}{4 \pi \epsilon_{o}}\left[\frac{\hat{\mathbf{n}}-\boldsymbol{\beta}}{\gamma^{2}(1-\boldsymbol{\beta} \cdot \hat{\mathbf{n}})^{3} R^{2}}\right]_{r e t}
$$

the transverse component of the electric field, in the unit system SI, at a constant hight $y=b$, with the beam extending along the $Z$-direction, considering that the point $M$ is the origin of the axes ([12], figure 14.2, page 664), is given by the equation:

$$
E_{2}=\frac{e}{4 \pi \epsilon_{o}} \frac{b}{\gamma^{2}\left(\sqrt{b^{2}+z^{2}}+\beta z\right)^{3}}
$$

and the maximum transverse component of the electric field is:

$$
E_{2 \max }=\frac{e}{4 \pi \epsilon_{o}} \frac{\gamma}{b^{2}}
$$

We first assume that a free electron beam propagates in a linear range of about 1000 meters, with the sensor in the middle of this distance. In Figure 2 is shown the curve $E_{2} / E_{2 \max }$, in such a lab, according to the previous analysis in [12].

Concerning the electron beam of the accelerator it is mentioned "... the 


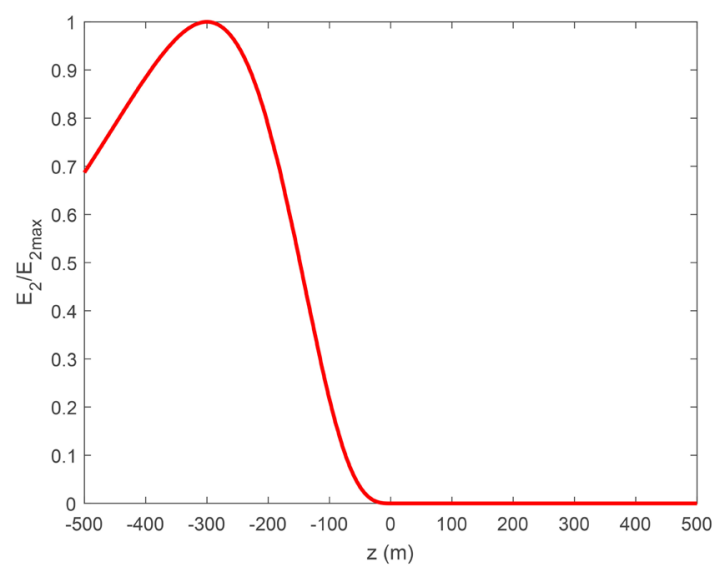

Figure 2. The curve $E_{2} / E_{2 \max }$ for a free electron beam propagating in a linear range of about 1000 meters.

electron beam produced at the DAФNE Beam Test Facility (BTF) [11], a beam line built and operated at the Frascati National Laboratory to produce a well-defined number of electrons (or positrons) with energies between $50 \mathrm{MeV}$ and $800 \mathrm{MeV}$." and its description is given in the phrase "At maximum intensity the facility yields, at a $50 \mathrm{~Hz}$ repetition rate, $10 \mathrm{~ns}$ long beams with a total charge up to several hundreds pCoulomb.".

Therefore the length of a portion of the beam coming from a burst is $\ell=10 \mathrm{~ns} \times u \simeq 3 \mathrm{~m}$. We assume that the sensor is above the middle of this segment and that $\phi$ is the angle formed by $\boldsymbol{R}\left(t^{\prime}\right)$ defining the position of the electron giving the signal, and by the linear segment $O M$ ([12], figure 14.2, page 664). The point $O$ is assumed at the bottom of the sensor.

According to the relation $R\left(t^{\prime}\right)-(1 / c) \boldsymbol{R}\left(t^{\prime}\right) \cdot \boldsymbol{u}=R(t) \sqrt{1-\left(u^{2} / c^{2}\right) \sin ^{2}(\theta(t))}$ ([13], \$63) and the relation $\boldsymbol{R}\left(t^{\prime}\right)-(\mathbf{u} / c) R\left(t^{\prime}\right)=\boldsymbol{R}(t)$ the electric field as a function of observation time $t$ is:

$$
\boldsymbol{E}(t)=\frac{e}{4 \pi \epsilon_{o}} \frac{\boldsymbol{R}(t)}{R(t)^{3}} \frac{1-u^{2} / c^{2}}{\left(1-\frac{u^{2}}{c^{2}} \sin ^{2}(\theta(t))\right)^{\frac{3}{2}}}
$$

The differential component derived from an elementary charge $\mathrm{d} q=\lambda \mathrm{d} z$, where $\lambda$ is the linear charge density of the segment of the beam in time $t$, taking into account that $y=b=R(t) \cos (\phi(t))$, is:

$$
\mathrm{d} E_{y}(t)=\frac{\lambda}{4 \pi \epsilon_{0} \gamma^{2}} \frac{\cos (\phi(t)) \mathrm{d} z}{R(t)^{2}\left(1-\frac{u^{2}}{c^{2}} \sin ^{2}(\theta(t))\right)^{\frac{3}{2}}}
$$

Since $R(t) \cos (\phi(t))=y$ and $\mathrm{d} \tan \phi(t)=\mathrm{d} z(t) / y=\mathrm{d} \phi(t) / \cos ^{2}(\phi(t))$, the following relation applies:

$$
\frac{\mathrm{d} z(t)}{R(t)^{2}}=\frac{\mathrm{d} \phi(t)}{y}
$$


The angles $\phi$ and $\theta$ are complementary, so:

$$
\mathrm{d} E_{y}(t)=\frac{\lambda}{4 \pi \epsilon_{o} \gamma^{2} y} \frac{\mathrm{d} \sin \phi}{\left(\frac{1}{\gamma^{2}}+\frac{u^{2}}{c^{2}} \sin ^{2}(\phi)\right)^{\frac{3}{2}}}
$$

For convenience, we set $\beta \sin \phi=x$, where $\beta=u / c$, and so:

$$
\mathrm{d} E_{y}(t)=\frac{\lambda}{4 \pi \epsilon_{o} \gamma^{2} y} \frac{1}{\beta} \frac{\mathrm{d} x}{\left(\frac{1}{\gamma^{2}}+x^{2}\right)^{\frac{3}{2}}}
$$

In a linear infinite distribution, because of the symmetry, the integration gives a transverse component of the electric field, at a height $y$, at time $t$, equal to:

$$
E_{y}(t)=\frac{2 \lambda}{4 \pi \epsilon_{o} \gamma^{2} y} \frac{1}{\beta} \int_{0}^{\beta} \frac{\mathrm{d} x}{\left(\frac{1}{\gamma^{2}}+x^{2}\right)^{\frac{3}{2}}}=\frac{\lambda}{2 \pi \epsilon_{o} \gamma^{2} y} \frac{1}{\beta}\left[\frac{x}{\frac{1}{\gamma^{2}} \sqrt{x^{2}+\frac{1}{\gamma^{2}}}}\right]_{0}^{\beta}=\frac{\lambda}{2 \pi \epsilon_{o} y}
$$

Since the length of this beam segment is finite $(\ell \simeq 3 \mathrm{~m}$ ), the maximum angle $\phi_{\max }$ is $79^{\circ}$ and the corresponding sine, at a height of $y=30 \mathrm{~cm}$, is $\sin \phi_{\max }=0.98$. Therefore, the term in square brackets, of the previous relation, becomes:

$$
\frac{1}{\beta}\left[\frac{x}{\frac{1}{\gamma^{2}} \sqrt{x^{2}+\frac{1}{\gamma^{2}}}}\right]_{0}^{0.98 \beta} \simeq 0.99999998 \gamma^{2} \simeq \gamma^{2}
$$

Therefore, this finite length portion of the beam gives a transverse electric field component equal to that which is given by an infinite length beam.

The maximum potential difference at the ends of the sensor $y_{1}=y, y_{2}=y+14 \mathrm{~cm}$ is:

$$
V_{\max }=\frac{\lambda}{2 \pi \epsilon_{o}} \int_{y_{1}}^{y_{2}} \frac{\mathrm{d} y}{y}=\frac{\lambda}{2 \pi \epsilon_{o}} \ln \left(\frac{y+14 \mathrm{~cm}}{y}\right)
$$

If we multiply the second member of the previous relationship by a calibration coefficient $\eta$, we get the relation (8) of the bibliographic reference [2].

The transverse component of the electric field derived from a single electron, based on the Equation (15), and the relation $R(t)=y / \sin \theta$, is given by the relation:

$$
E_{2}(t)=\frac{e}{4 \pi \epsilon_{o}} \frac{\sin ^{3} \theta}{y^{2}} \frac{1-u^{2} / c^{2}}{\left(1-\frac{u^{2}}{c^{2}} \sin ^{2}(\theta(t))\right)^{\frac{3}{2}}}
$$

The ratio $E_{2}(t) / E_{2 \max }$ is therefore given by the relation: 


$$
\frac{E_{2}(t)}{E_{2 \max }}=\frac{\sin ^{3}(\theta(t))}{\gamma^{3}\left(1-\frac{u^{2}}{c^{2}} \sin ^{2}(\theta(t))\right)^{\frac{3}{2}}}
$$

In Figure 3 is shown the graph of the function $E_{2}(t) / E_{2 \max }$ of the previous relation. This function looks like a delta function.

As can be seen from Figure 3 only the electrons beneath the sensor, at a very short distance from the point $M$, contribute in the transverse component of the electric field. This means that the corresponding signals depart from a position $Z$ near the position $z=-300 \mathrm{~m}$ in the negative semi axis.

In order to better understand this, we will calculate the relation between $z\left(t^{\prime}\right)$ and $z_{t}=z(t)$. From the known relation $z(t)=z\left(t^{\prime}\right)+\beta R\left(t^{\prime}\right)=z\left(t^{\prime}\right)+\beta \sqrt{b^{2}+z\left(t^{\prime}\right)^{2}}$, by setting $z=z\left(t^{\prime}\right), \quad z_{t}=z(t)$, we obtain the following equation:

$$
z^{2}-2 \gamma^{2} z_{t} z+\gamma^{2} z_{t}^{2}-\beta^{2} \gamma^{2} b^{2}=0
$$

The resulting values of $z$ are $z=\gamma^{2} z_{t} \pm \beta \gamma \sqrt{\gamma^{2} z_{t}^{2}+b^{2}}$. The value with (+) is rejected, since for $z_{t}=0$ we have the relation $z=-\beta \gamma b$. This solution shows that for very short distances from $M$ we have large absolute values of $z$ and that is why we take the curve of Figure 2.

However, since in the laboratory of this experiment the electronic beam extends at distances of less than $10 \mathrm{~m}$, the sensor should receive signals departing from this region, and so the transverse component of the electric field should be much smaller relative to the maximum we calculated in the previous relation.

In Figure 4 is shown the graph of the ratio $E_{2} / E_{2 \max }$ in the range $-10 \mathrm{~m}<\mathrm{z}<0$. It is obvious that the ratio $E_{2} / E_{2 \max }$ in the range $-10 \mathrm{~m}<\mathrm{z}<0$ is of the order of $10^{-4}$, and such a signal is not detectable by the experimental device. Therefore, the measured signal is many orders of magnitude larger than the predicted by relativistic theory.

From the Equation (25), if we consider $b=y$, we obtain an acceptable solution $z_{t}=z+\beta \sqrt{z^{2}+y^{2}}$. Assuming that the left end of the portion of the electron beam is in the initial position where the charges generating the field are not be shielded by conductors and it is possible to emit an electromagnetic signal, then the signal will arrive at the sensor at time $t$ and this end of the beam will be at the corresponding position $z_{t 1}(t)$. The right end of the beam at the same time $t$ will be at the position $z_{t 2}=z_{t 1}+3 \mathrm{~m}$. The limits of integration of the relation (20) are determined by the relation $x_{i}=\beta \sin \left(\arctan \left(z_{t i} / y\right)\right)$.

The calculation of the ratio of the relativistic maximum electrical potential differences to measured maximum electrical potential at three different positions of the sensor in the direction of $Z$, at $1720 \mathrm{~mm}, 3295 \mathrm{~mm}$, and $5525 \mathrm{~mm}$, but at a constant height from the lower end of sensor end at $30 \mathrm{~cm}$, gives us:

$$
\begin{aligned}
& \frac{V_{1720}}{V_{\text {max }}}=4.6914 \mathrm{e}-05 \\
& \frac{V_{3295}}{V_{\text {max }}}=1.6953 \mathrm{e}-04
\end{aligned}
$$




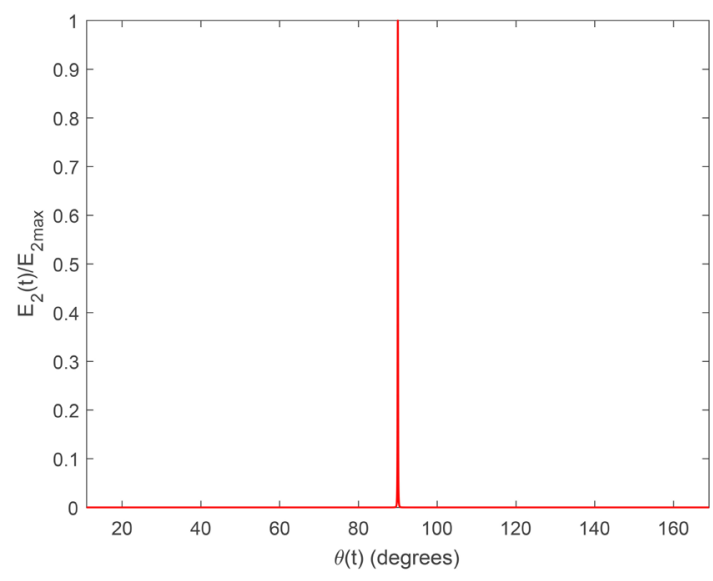

Figure 3. The curve of the function $E_{2} / E_{2 \max }$ in time $t$ looks like a delta function.

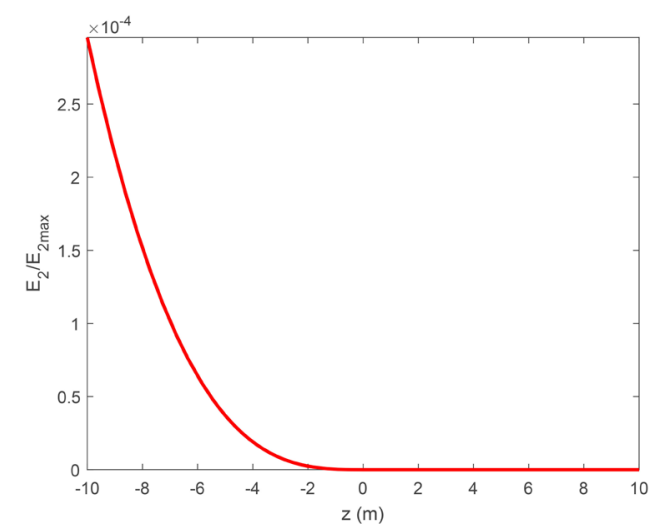

Figure 4. The ratio $E_{2} / E_{2 \max }$ in the range $-10 \mathrm{~m}<\mathrm{z}<0$ is of the order of $10^{-4}$.

$$
\frac{V_{5525}}{V_{\max }}=4.7477 \mathrm{e}-04
$$

where $V_{1720}, V_{3295}, V_{5525}$, the calculated electrical potential differences according to the special theory of relativity, that is, at the height $y=30 \mathrm{~cm}$ at which we consider that is the location of the sensor, the measured $V_{\max }$ is many orders of magnitude higher than the one would expect from the calculations of relativistic electric potential differences.

In Figure 5 is shown the graph of the ratio $V_{\text {rel }}(t) / V_{\text {max }}$ versus time, within time interval $-10 \mathrm{~ns}<t<20 \mathrm{~ns}$, at $z_{A 5, A 6}=1.72 \mathrm{~m}$, transverse distance $30 \mathrm{~cm}$. We consider that $t=0$ is the time the initial signal is received at the sensor.

However, the measurements of this experiment show that the maximum electric potential differences are in agreement with the relation (22). Therefore, as shown in Figure 5, at $Z_{A 5, A 6}=1.72 \mathrm{~m}$, transverse distance $30 \mathrm{~cm}$, given that $V_{1720} / V_{\max }=4.6914 \mathrm{e}-05$ according to previous calculations, the highest value of the relativistic potential difference is about 20,000 times smaller than the one measured in the experiment. 


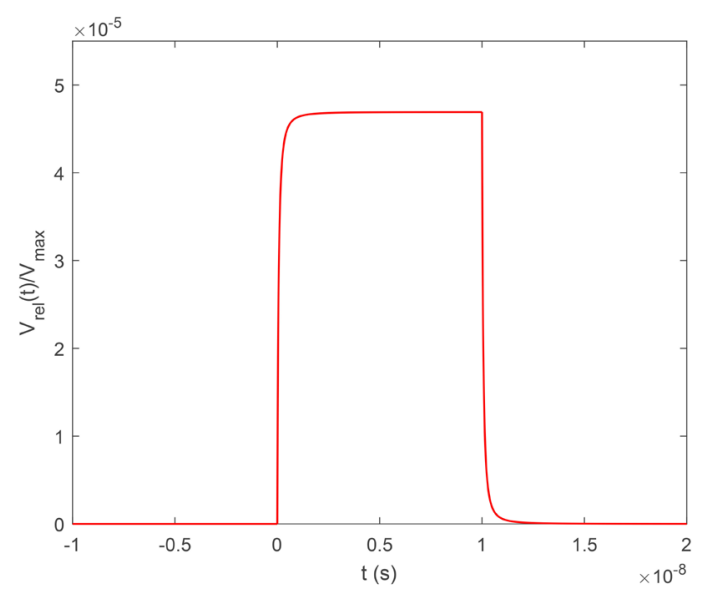

Figure 5. The ratio $V_{\text {rel }}(t) / V_{\max }$ within time $-10 \mathrm{~ns}<t<20 \mathrm{~ns}$

at $Z_{A 5, A 6}=1.72 \mathrm{~m}$, transverse distance $30 \mathrm{~cm}$.

\section{Theoretical Study According to the Hypothesis of the Absolute Reference System}

In the abstract of my published work entitled "The Physics of an Absolute Reference System" ([3] [4]), the first of the basic principles of the absolute reference system hypothesis is described as "... The first of these principles is that the electromagnetic field quantitative estimates are made in the inertial reference system of the source of the electromagnetic field ..." Also, according to the hypothesis of the absolute reference system, there are no transformations of physical magnitudes, because the Lorentz contraction is physical, not geometric, that is, it is not a space-time view of the observer, as in the special theory of relativity.

In the present experiment of the reference [2] the inertial reference systems are two, namely the first one is the reference system of the laboratory and the other is that of the electronic beam. Therefore, because the source of the electric field is the beam electrons, the electric field may be calculated in the inertial reference system of the electron beam and this calculated value is equal to the calculated value of the electric field in the reference system of laboratory. This principle contradicts the basic principles of special theory of relativity, since it does not take into account the relative velocity between the beam electrons and the sensor, but only the speed of the beam inertial system relative to the inertial reference system of laboratory (because the inertial system of the beam comes from the action of electromagnetic fields whose sources are in the laboratory). This means that according to the hypothesis of the absolute reference system there is no the relativistic concept of the evaluation of the electric field at the retarded time giving the corresponding electrical and magnetic potential at the observation point. Also in the beam reference system, the sensor "scans" the electric field region, while in the laboratory reference system there is no the limitation of the maximum velocity $c$ at the speed of the interaction photons. Therefore under this condition, an observer of the laboratory will see an electric field rigidly carried by the beam itself. 
We will now calculate the electric field derived from a portion of the electron beam with a linear density $\lambda$ in the beam reference system, according to the hypothesis of the absolute reference system. The maximum value of the calculated electric field at a height of $y$, at the observation point, that is, at the sensor, will be when the sensor is above the mean of the linear charge distribution. Since the sensor is stationary in the laboratory, the calculation of electric field is derived from Maxwell's equations. So, it is calculated according to the relation:

$$
\boldsymbol{E}_{M}=\frac{\lambda}{2 \pi \epsilon_{o} y} \hat{\boldsymbol{j}}
$$

where the index $M$ means that the electric field is calculated according to Maxwell's equations and $\hat{\boldsymbol{j}}$ is a unit vector in the $Y$-direction.

The electric potential difference between the $y_{1}$ and $y_{2}$ sensor edges is calculated as follows:

$$
V_{\max }=\frac{\lambda}{2 \pi \epsilon_{o}}[\ln y]_{y_{1}}^{y_{2}}
$$

so for $y_{1}=y$ and $y_{2}=y+14 \mathrm{~cm}$ this relation becomes:

$$
V_{\max }=\frac{\lambda}{2 \pi \epsilon_{o}} \ln \left(\frac{y+14 \mathrm{~cm}}{y}\right)
$$

\section{Positron-Electron Annihilation Process}

An experiment implemented in order to experimentally investigate relativistic kinematics in an undergraduate student laboratory is the article in [14], and it is showed that it is possible to use the flight positron annihilation for the experimentally study of the special relativistic kinematic relation between momentum and energy.

In this article we give the theoretical background for this process using the kinematics of the hypothesis of the absolute reference system. We consider a positron with kinetic energy $E$ and momentum $\boldsymbol{p}$ that annihilates with an electron at rest with the emission of two photons as shown in Figure 6. The energy and the momentum of the emitted photons are $E_{1}, \boldsymbol{p}_{1}$ and $E_{2}, \boldsymbol{p}_{2}$. The energy momentum relations of the emitted photons are $E_{1}=p_{1} c=h v_{1}$ and $E_{2}=p_{2} c=h v_{2}$, where $v_{1}, v_{2}$ are the frequencies of them and the corresponding kinetic energies are $\frac{1}{2} h v_{1}=\frac{1}{2} E_{1}, \frac{1}{2} h v_{2}=\frac{1}{2} E_{2}$.

The total kinetic energy of the positron is $E=(1 / 2) m \gamma^{2} c^{2}$ and because the frequencies of the bound photons measured with the clock of the inertial reference system of the laboratory are $\gamma$ times smaller than the corresponding ones measured in the reference system of the positron, the contribution of positron to energy of the emitted photons is equal to $E_{l}=E / \gamma=(1 / 2) m \gamma c^{2}$, while the total kinetic energy of the electron that is at rest in the laboratory is equal to $(1 / 2) m c^{2}$. Conservation of momentum and energy give 


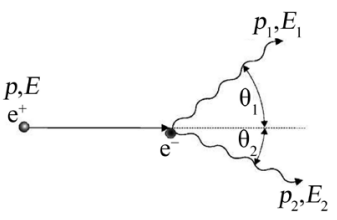

Figure 6. Energy and momentum of the positron-electron annihilation in flight.

$$
\begin{gathered}
\boldsymbol{p}=\boldsymbol{p}_{1}+\boldsymbol{p}_{2} \\
E_{l}+\frac{1}{2} m c^{2}=\frac{1}{2} E_{1}+\frac{1}{2} E_{2}
\end{gathered}
$$

Squaring both sides of the Equation (29) we get

$$
p^{2}=p_{1}^{2}+p_{2}^{2}+2 p_{1} p_{2} \cos \theta
$$

Using the previous relations and the relation $\left(m \gamma c^{2}\right)^{2}=p^{2} c^{2}+m^{2} c^{4}$, which is valid in relativistic kinematics and in kinematics of hypothesis of absolute reference system, and after some simple algebraic calculations, we get the following relation,

$$
\frac{1}{E_{1}}+\frac{1}{E_{2}}=\frac{1-\cos \theta}{m c^{2}}
$$

which is the same as that derived from relativistic kinematics. Also, the last equation is the main result that was experimentally verified in [14].

\section{Conclusion}

In all experiments studied in this article on the basis of the hypothesis of an absolute reference system, it appeared unequivocally that the experimental results are in excellent agreement with the theoretically predicted results of this hypothesis. There are further similar issues to be discussed, but the most of them have already been discussed in the initial publications of the hypothesis of the absolute reference system. In the present study some issues discussed in order to test the validity of this hypothesis. However, as much as possible experimental research is suggested, mainly taking as many as possible experimental data on the speed and energy values of the Bertozzi's experiment, as the results of this experiment have a one-sided interpretation in the scientific world, in order for us to reach scientific conclusions, fully accepted by the scientific community, regarding to the agreement of the hypothesis of absolute reference system with this experiment.

\section{Conflicts of Interest}

The author declares no conflicts of interest regarding the publication of this paper.

\section{References}

[1] Feynman, R.P., Leighton, R.B. and Sands, M. (1977) The Feynman Lectures on 
Physics, Volume II, Mainly Electromagnetism and Matter. California Institute of Technology, Pasadena.

[2] de Sangro, R., Finocchiaro, G., Patteri, P., Piccollo, M. and Pizzella, G. (2015) Measuring Propagation Speed of Coulomb Fields. The European Physical Journal C, 75, 137. https://doi.org/10.1140/epjc/s10052-015-3355-3

[3] Patrinos, K. (2019) The Physics of an Absolute Reference System. Journal of Applied Mathematics and Physics, 7, 431-475. https://doi.org/10.4236/jamp.2019.73033

[4] Patrinos, K. (2019) Classical Quantum Field Theory Based on the Hypothesis of the Absolute Reference System. Journal of Applied Mathematics and Physics, 7, 747-780. https://doi.org/10.4236/jamp.2019.74052

[5] Kündig, W. (1963) Measurement of the Transverse Doppler Effect in an Accelerated System. The Physical Review, 129, 2371. https://doi.org/10.1103/PhysRev.129.2371

[6] Kholmetskii, A.L., Yarman, T. and Missevitch, O.V. (2008) Kündig's Experiment on the Transverse Doppler Shift Re-Analyzed. Physica Scripta, 77, Article ID: 035302. https://doi.org/10.1088/0031-8949/77/03/035302

[7] Kholmetskii, A.L., Yarman, T., Missevitch, O.V. and Rogozev, B.I. (2009) A Mössbauer Experiment in a Rotating System on the Second-Order Doppler Shift: Confirmation of the Corrected Result by Kündig. Physica Scripta, 79, Article ID: 065007. https://doi.org/10.1088/0031-8949/79/06/065007

[8] Corda, C. (2015) Interpretation of Mössbauer Experiment in a Rotating System: A New Proof for General Relativity. Annals of Physics, 355, 360-366. https://doi.org/10.1016/j.aop.2015.02.021

[9] Kholmetskii, A.L., Yarmanb, T. and Arik, M. (2015) Comment on "Interpretation of Mössbauer Experiment in a Rotating System: A New Proof by General Relativity”. Annals of Physics, 363, 556-558. https://doi.org/10.1016/j.aop.2015.09.007

[10] Bertozzi, W. (1964) Education Development Center, Newton, Mass. 1962? The Ultimate Speed? American Journal of Physics, 32, 551-555. https://doi.org/10.1119/1.1970770

[11] Lund, M. and Uggerhøj, U.I. (2009) Experimental Special Relativity with a Meter Stick and a Clock. American Journal of Physics, 77, 757. https://doi.org/10.1119/1.3049532

[12] Jackson, J.D. (1998) Classical Electrodynamics. 3rd Edition, John Wiley \& Sons, Hoboken.

[13] Landau, L.D. and Lifshitz, E.M. (1971) The Classical Theory of Fields. Third Revised English Edition, Pergamon Press, Oxford, New York, Toronto.

[14] Dryzek, J., Singleton, D., Suzuki, T. and Yu, R. (2006) An Undergraduate Experiment to Test Relativistic Kinematics Using in Flight Positron Annihilation. American Journal of Physics, 74, 49. https://doi.org/10.1119/1.2142624 